\title{
A new functional group selection method for group contribution models and its application in the design of electronics cooling fluids
}

\author{
Yijia $\operatorname{Sun}^{1}$ and Nikolaos V. Sahinidis ${ }^{1,2,3}$, \\ ${ }^{1}$ Department of Chemical Engineering, Carnegie Mellon \\ University, Pittsburgh, PA, 15213, United States
}

${ }^{2}$ H. Milton Stewart School of Industrial \& Systems Engineering, Georgia Institute of Technology, Atlanta, GA, 30332, United States

${ }^{3}$ School of Chemical \& Biomolecular Engineering, Georgia Institute of Technology, Atlanta, GA, 30332, United States 


\section{Supporting Information for Publication}

\section{Fitting correlation plots and error residual histograms}
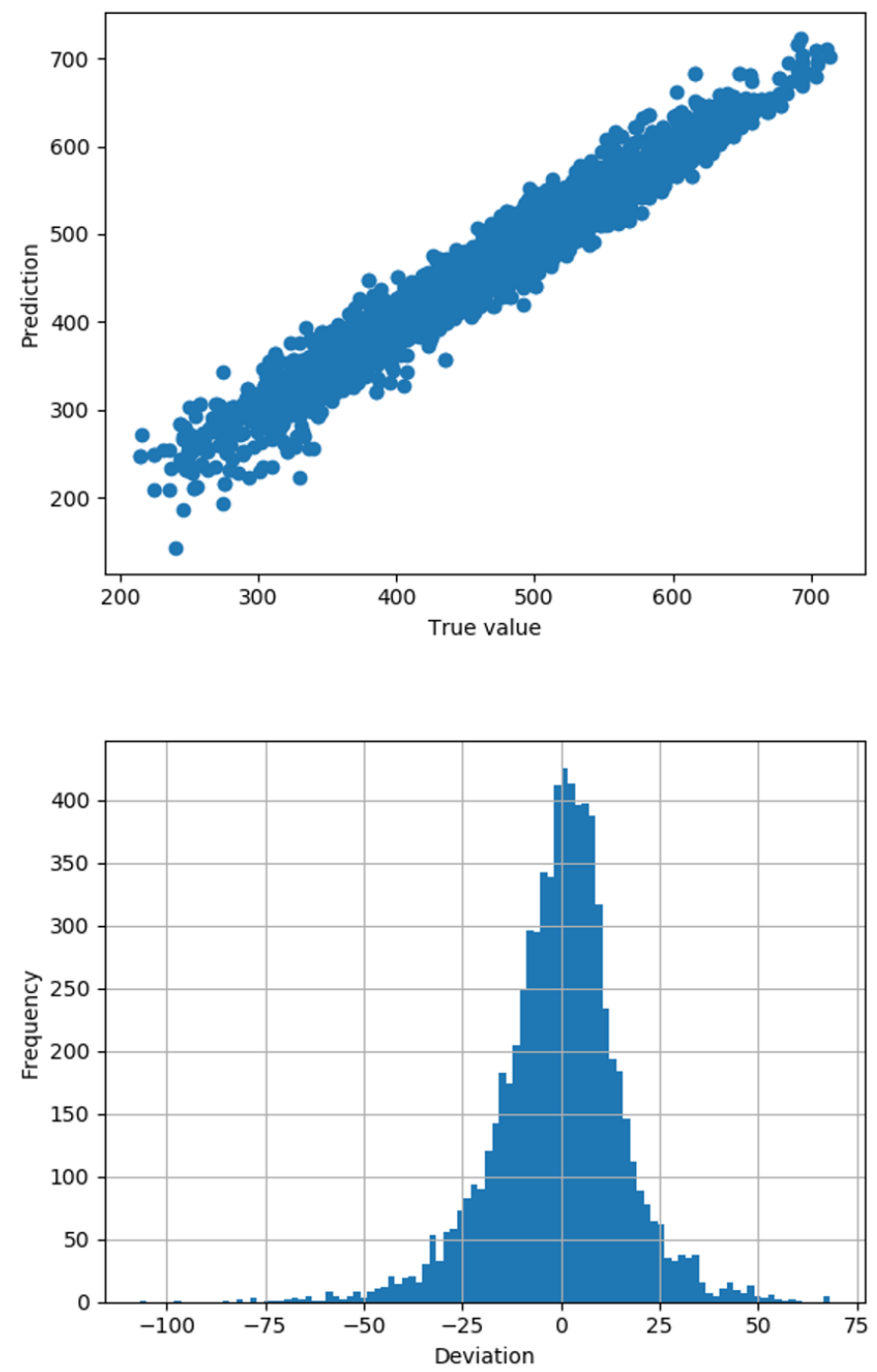

Figure S1. Normal boiling point (K) parity plot $\left(R^{2}=0.96\right)$ and error residual plot. $75.27 \%$ of the compounds deviate by no more than one training RMSE. 

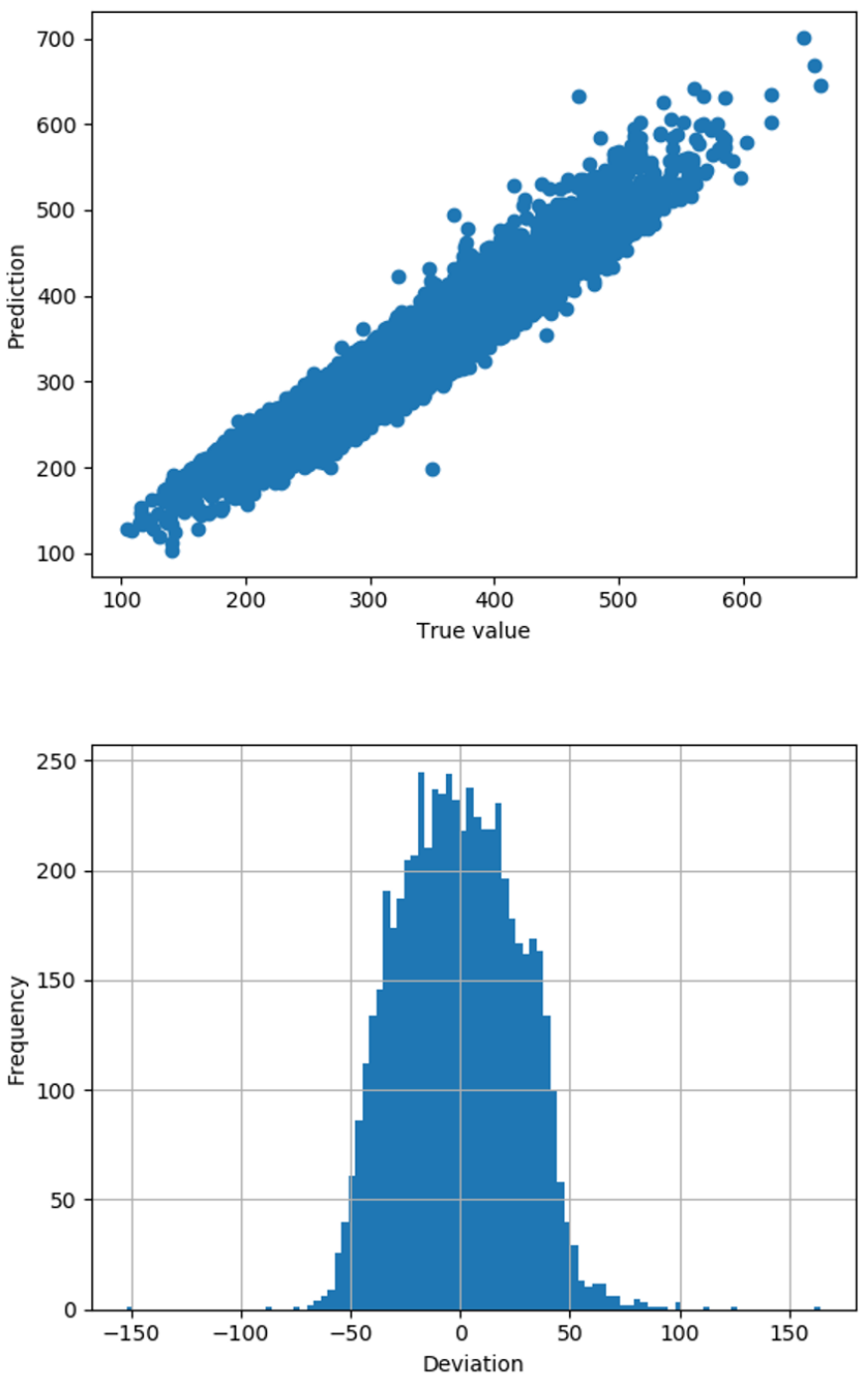

Figure S2. Melting point $(K)$ parity plot $\left(R^{2}=0.90\right)$ and error residual plot. $64.04 \%$ of the compounds deviate by no more than one training RMSE. 

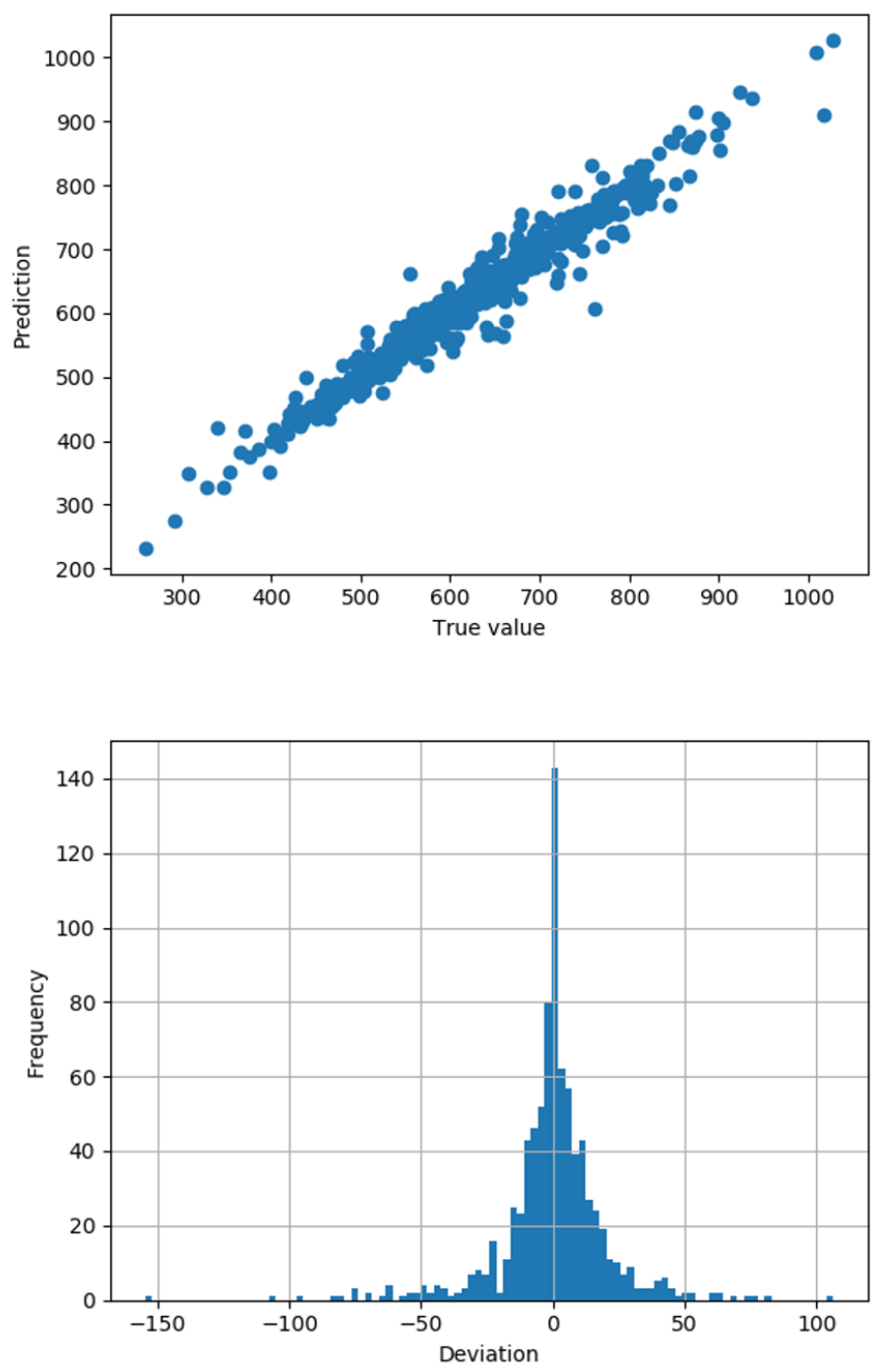

Figure S3. Critical point $(K)$ parity plot $\left(R^{2}=0.96\right)$ and error residual plot. $81.94 \%$ of the compounds deviate by no more than one training RMSE. 

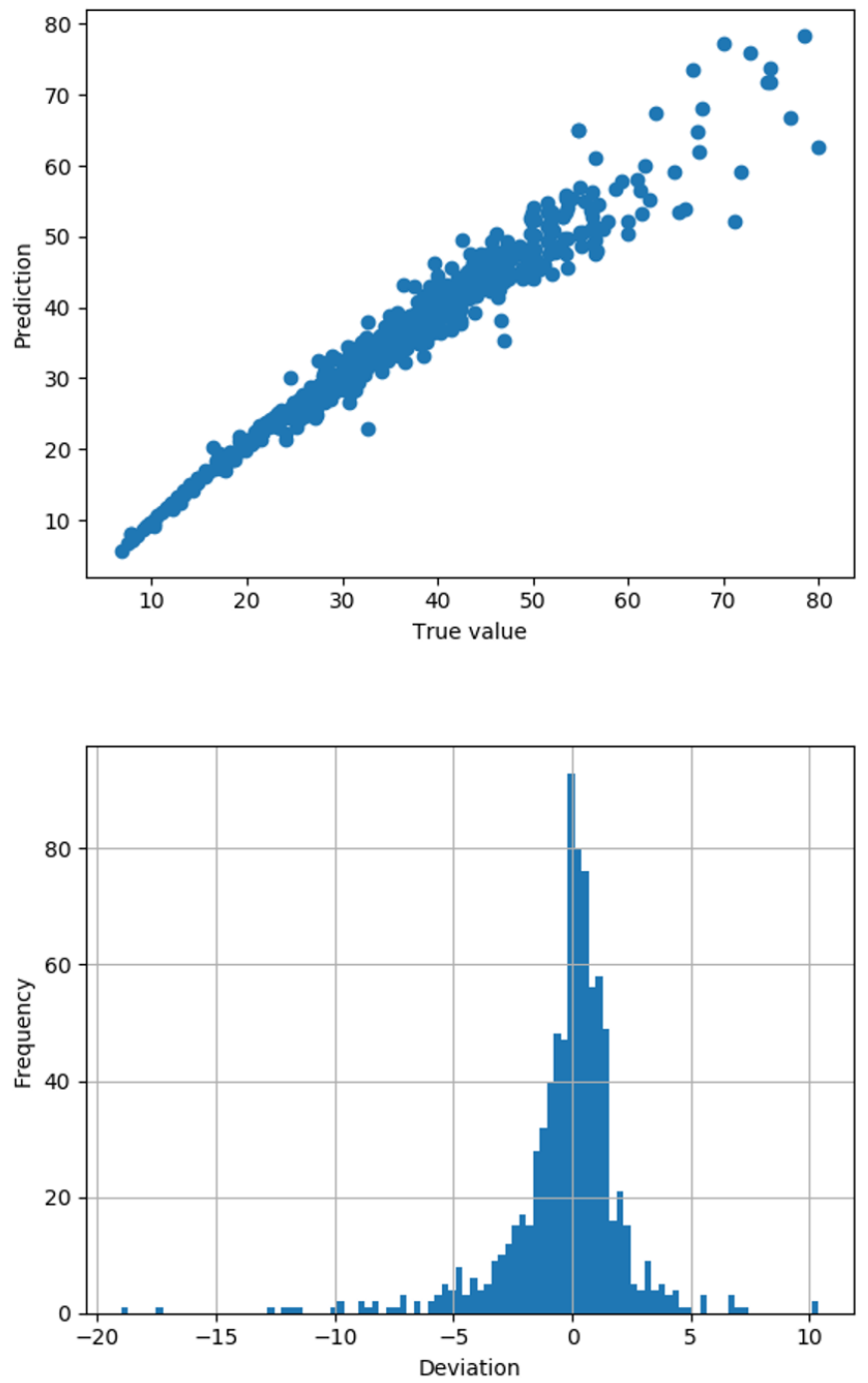

Figure S4. Critical pressure (bar) parity plot $\left(\mathrm{R}^{2}=0.95\right)$ and error residual plot. $83.17 \%$ of the compounds deviate by no more than one training RMSE. 

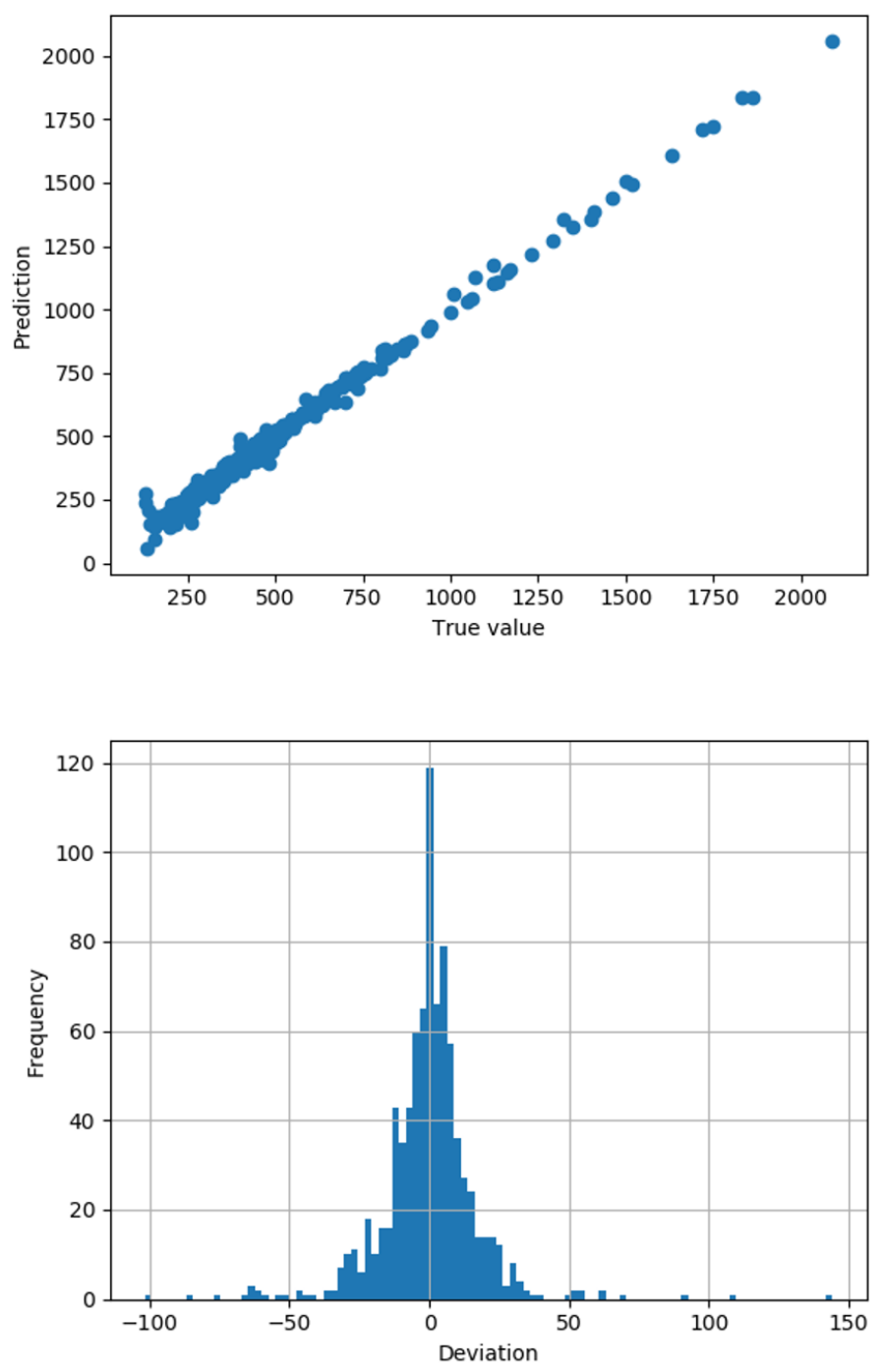

Figure S5. Critical volume $(\mathrm{cc} / \mathrm{mol})$ parity plot $\left(R^{2}=0.99\right)$ and error residual plot. $80.75 \%$ of the compounds deviate by no more than one training RMSE. 

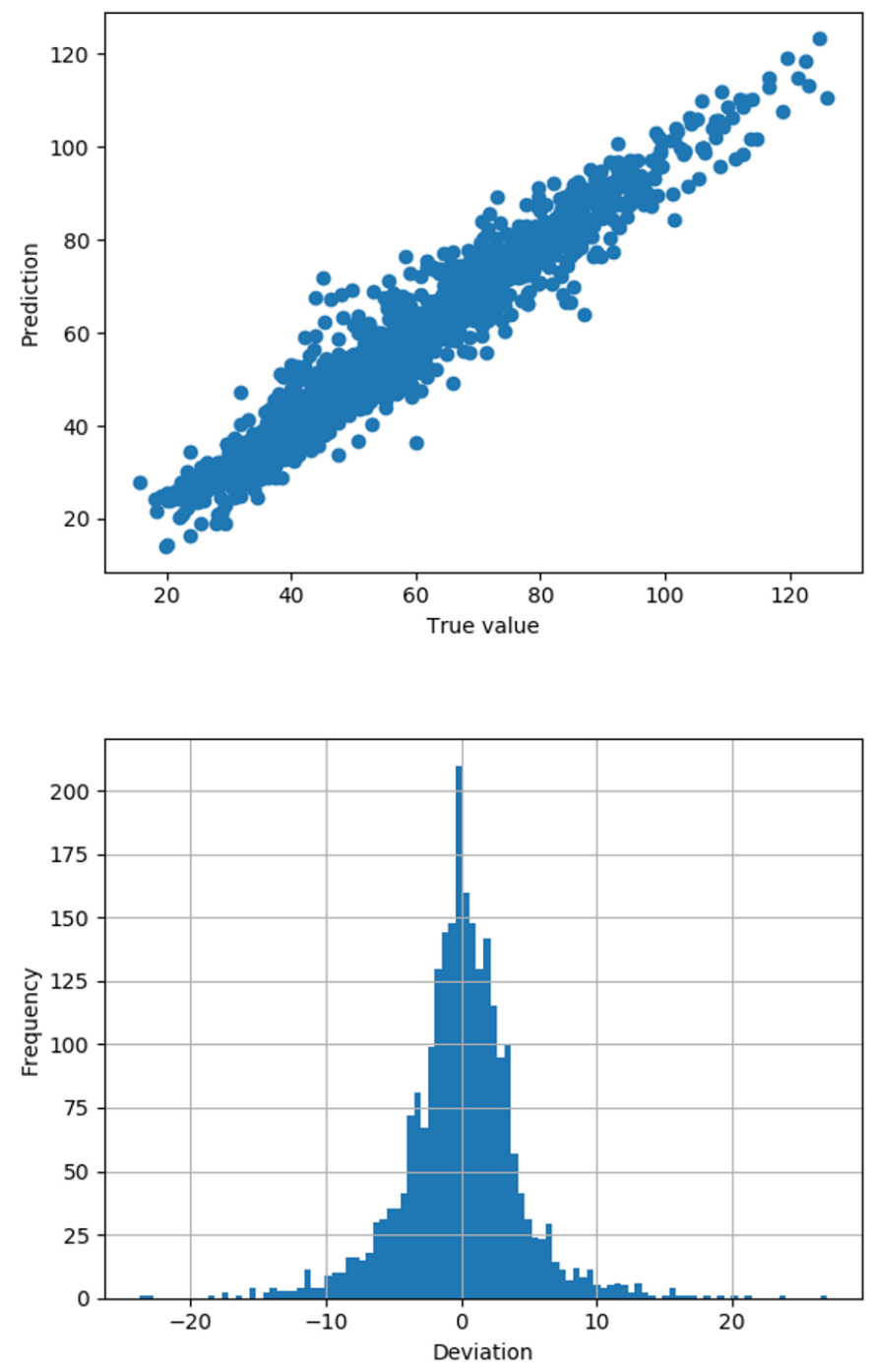

Figure S6. Enthalpy of vaporization at $298 \mathrm{~K}(\mathrm{~kJ} / \mathrm{mol})$ parity plot $\left(\mathrm{R}^{2}=0.95\right)$ and error residual plot. $78.33 \%$ of the compounds deviate by no more than one training RMSE. 

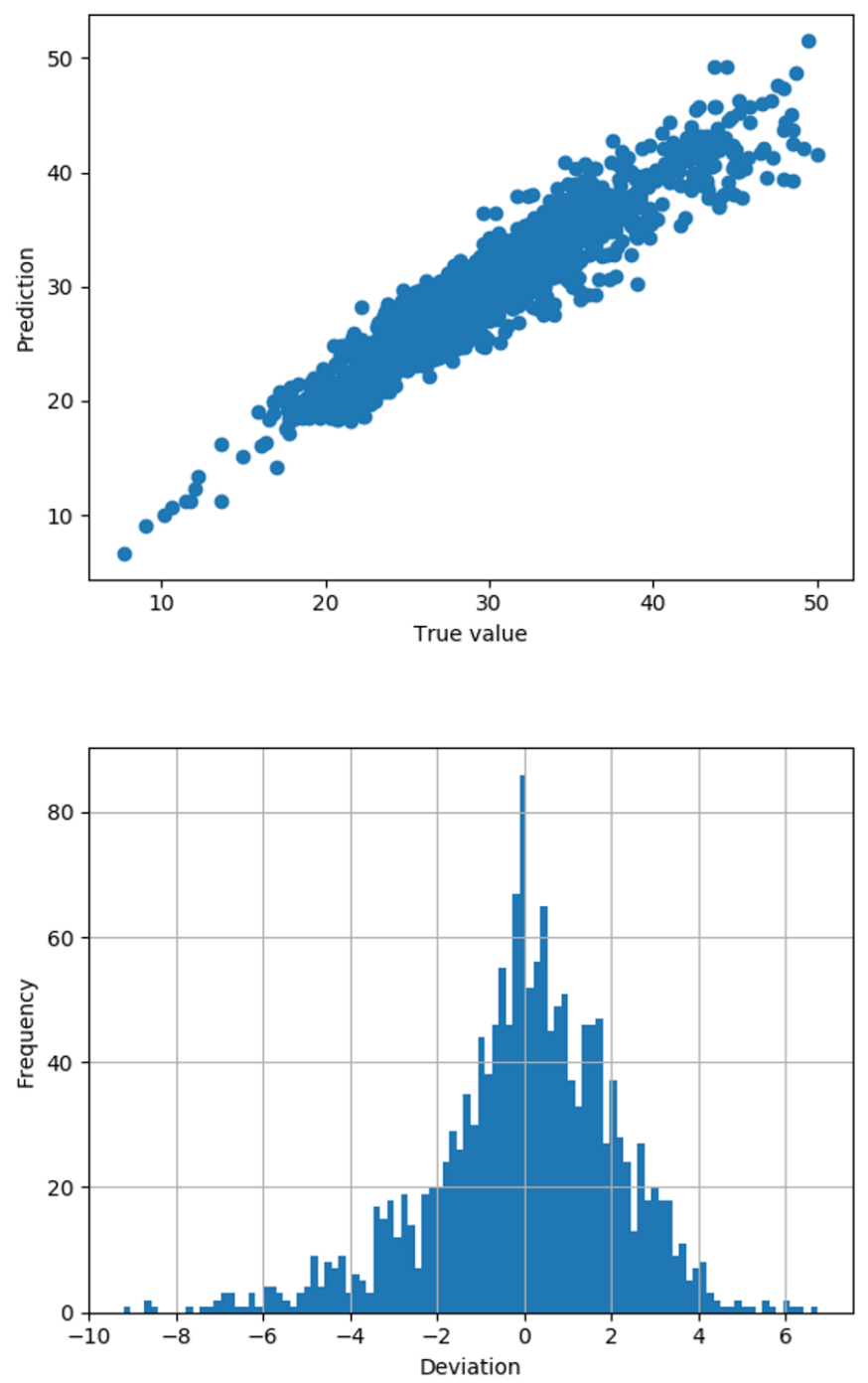

Figure S7. Surface tension $(\mathrm{N} / \mathrm{m})$ parity plot $\left(\mathrm{R}^{2}=0.89\right)$ and error residual plot. $72.38 \%$ of the compounds deviate by no more than one training RMSE. 

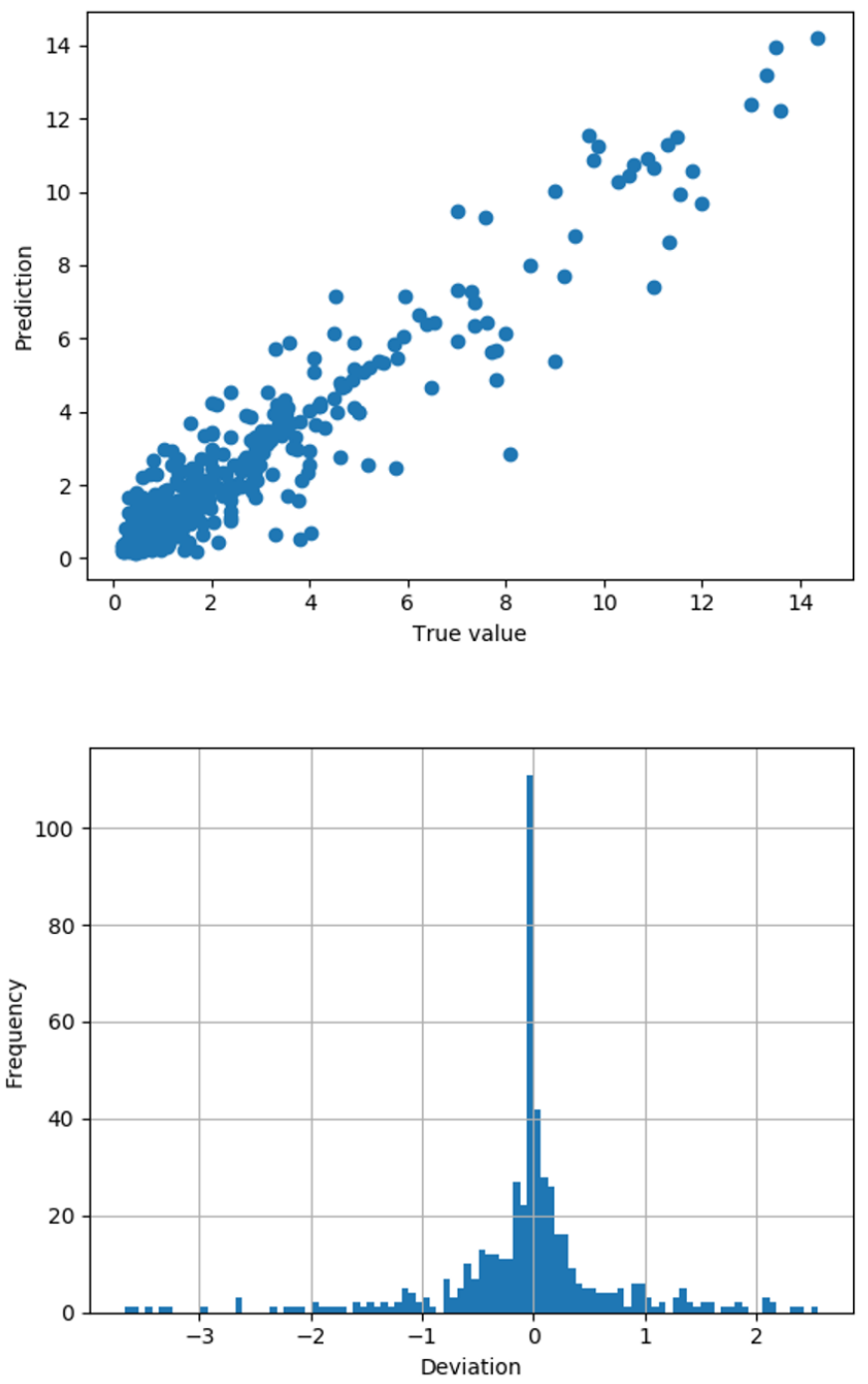

Figure S8. Dynamic viscosity (mPa s) parity plot $\left(\mathrm{R}^{2}=0.91\right)$ and error residual plot. $81.87 \%$ of the compounds deviate by no more than one training RMSE. 


\section{Target property calculations of the best performing organosilicon and non-organosilicon coolants}

Table S1. Target property calculations of top-10 organosilicon candidates

\begin{tabular}{ccccc}
\hline SMILES & $\mathbf{T}_{\mathbf{b}}(\mathbf{K})$ & $\mathbf{T}_{\mathbf{m}}(\mathbf{K})$ & $\mathbf{H}_{\mathbf{v a p}}(\mathbf{K J} \mathbf{j} \mathbf{m o l})$ & $\boldsymbol{\mu}(\mathbf{m P a} \mathbf{~})$ \\
\hline $\mathrm{CCO}[\mathrm{Si}] \mathrm{C}(\mathrm{C}) \mathrm{CC}$ & 417.60 & 202.92 & 41.16 & 0.51 \\
\hline $\mathrm{COC}(\mathrm{C}) \mathrm{OC}(\mathrm{C})[\mathrm{Si}] \mathrm{C}$ & 420.05 & 203.37 & 54.9 & 0.59 \\
\hline $\mathrm{COC}(\mathrm{C})[\mathrm{Si}] \mathrm{C}(\mathrm{C}) \mathrm{OC}$ & 424.25 & 203.62 & 55.29 & 0.67 \\
\hline $\mathrm{CCC}[\mathrm{Si}] \mathrm{C}(\mathrm{C}) \mathrm{OC}$ & 415.70 & 212.86 & 53.45 & 0.58 \\
\hline $\mathrm{COCCC}(\mathrm{C})[\mathrm{Si}] \mathrm{C}$ & 421.64 & 203.73 & 42.5 & 0.62 \\
\hline $\mathrm{COCC}(\mathrm{OC})[\mathrm{Si}] \mathrm{C}$ & 423.26 & 210.69 & 43.11 & 0.70 \\
\hline $\mathrm{COC}[\mathrm{Si}] \mathrm{C}(\mathrm{C}) \mathrm{OC}$ & 417.36 & 220.39 & 54.06 & 0.60 \\
\hline $\mathrm{COC}(\mathrm{C}) \mathrm{OC}[\mathrm{Si}] \mathrm{C}$ & 420.25 & 220.47 & 54.16 & 0.68 \\
\hline $\mathrm{CO}[\mathrm{Si}] \mathrm{C}(\mathrm{C}) \mathrm{OC}$ & 388.12 & 217.24 & 49.01 & 0.56 \\
\hline $\mathrm{COC}(\mathrm{C}) \mathrm{O}[\mathrm{Si}] \mathrm{C}$ & 391.41 & 217.17 & 48.91 & 0.57 \\
\hline
\end{tabular}

Table S2. Target property calculations of top-10 non-organosilicon candidates

\begin{tabular}{ccccc}
\hline SMILES & $\mathbf{T}_{\mathbf{b}}(\mathbf{K})$ & $\mathbf{T}_{\mathbf{m}}(\mathbf{K})$ & $\mathbf{H}_{\mathbf{v a p}}(\mathbf{K J} \mathbf{j} \mathbf{m o l})$ & $\boldsymbol{\mu}(\mathbf{m P a} \mathbf{~})$ \\
\hline $\mathrm{FCCOC}(\mathrm{CC}(\mathrm{F})(\mathrm{F}) \mathrm{F})(\mathrm{F}) \mathrm{F}$ & 340.18 & 215.02 & 43.85 & 0.32 \\
\hline $\mathrm{C}=\mathrm{CC}(\mathrm{CCC}(\mathrm{F})(\mathrm{F}) \mathrm{F})(\mathrm{F}) \mathrm{F}$ & 339.29 & 179.37 & 34.56 & 0.32 \\
\hline $\mathrm{CCOC}(\mathrm{C}(\mathrm{C}(\mathrm{F})(\mathrm{F}) \mathrm{F}) \mathrm{C})(\mathrm{F}) \mathrm{F}$ & 341.31 & 186.56 & 39.1 & 0.38 \\
\hline $\mathrm{COCC}(\mathrm{C}(\mathrm{F})(\mathrm{F}) \mathrm{F}) \mathrm{C}(\mathrm{F})(\mathrm{F}) \mathrm{C}$ & 341.31 & 186.9 & 39.58 & 0.38 \\
\hline $\mathrm{CC}(\mathrm{COC}(\mathrm{C}(\mathrm{F})(\mathrm{F}) \mathrm{F})(\mathrm{F}) \mathrm{C})(\mathrm{F}) \mathrm{F}$ & 341.94 & 201.14 & 37.42 & 0.50 \\
\hline $\mathrm{CC}(=\mathrm{C}) \mathrm{C}(\mathrm{CC}(\mathrm{F})(\mathrm{F}) \mathrm{F})(\mathrm{F}) \mathrm{F}$ & 330.05 & 179.76 & 34.5 & 0.18 \\
\hline $\mathrm{CCC}(=\mathrm{CF}) \mathrm{CC}$ & 353.83 & 193.31 & 33.55 & 0.25 \\
\hline $\mathrm{C}=\mathrm{CC}(\mathrm{CC}(\mathrm{C}(\mathrm{F})(\mathrm{F}) \mathrm{F})(\mathrm{F}) \mathrm{F}) \mathrm{C}$ & 349.34 & 166.45 & 37.83 & 0.33 \\
\hline $\mathrm{CC}(\mathrm{C}(\mathrm{OC}(\mathrm{C}(\mathrm{F})(\mathrm{F}) \mathrm{F})(\mathrm{F}) \mathrm{F})(\mathrm{F}) \mathrm{F})(\mathrm{F}) \mathrm{F}$ & 308.14 & 188.04 & 33.76 & 0.39 \\
\hline $\mathrm{COC}(=\mathrm{O}) \mathrm{C}(\mathrm{C}(\mathrm{F})(\mathrm{F}) \mathrm{F})(\mathrm{F}) \mathrm{F}$ & 337.22 & 209.27 & 46.57 & 0.71
\end{tabular}

